\title{
CORRECTION
}

\section{Correction to: Treatment of aggressive adult T-cell leukemia/ lymphoma: a retrospective study in a hospital located in HTLV-1 highly endemic area}

\author{
Daisuke Nakamura ${ }^{1,2} \cdot$ Makoto Yoshimitsu $^{2} \cdot$ Tomohisa Tabuchi $^{1,2} \cdot$ Naosuke Arima $^{1,2} \cdot$ Maiko Hayashida $^{1,2}$. \\ Hirosaka Inoue ${ }^{1,2} \cdot$ Kakushi Matsushita $^{1,2} \cdot$ Tadashi Matsumoto $^{1} \cdot$ Naomichi Arima $^{2} \cdot$ Kenji Ishitsuka $^{2}$
}

Published online: 6 March 2020

(c) Japanese Society of Hematology 2020

\section{Correction to: \\ International Journal of Hematology (2020) 111:234-240 \\ https://doi.org/10.1007/s12185-019-02769-w}

In the original publication of the article, Table 5 was published with incorrect contents. The correct Table 5 is given in this correction.

Table 5 Relative dose intensity (RDI) of younger and elderly patients treated by OPEC/MPEC or VCAP-AMP-VECP chemotherapy

\begin{tabular}{lll}
\hline & RDI $(\%) \pm$ SD & $p$ value \\
\hline Patients aged $<70$ years & & \\
OPEC/MPEC $(n=58)$ & $89.5 \pm 17.1$ & 0.175 \\
VCAP-AMP-VECP $(n=20)$ & $83.7 \pm 12.9$ & \\
Patients aged $\geqq 70$ years & & \\
OPEC/MPEC $(n=80)$ & $88.6 \pm 18$ & 0.002 \\
VCAP-AMP-VECP $(n=13)$ & $71.6 \pm 14.8$ & \\
\hline
\end{tabular}

The original article can be found online at https://doi.org/10.1007/ s12185-019-02769-w.

Daisuke Nakamura

d5039@m3.kufm.kagoshima-u.ac.jp

Makoto Yoshimitsu

myoshimi@m.kufm.kagoshima-u.ac.jp

Tomohisa Tabuchi

tomohisatabuchi1116@gmail.com

Naosuke Arima

aritouma@m2.kufm.kagoshima-u.ac.jp

Maiko Hayashida

smarf@m3.kufm.kagoshima-u.ac.jp

Hirosaka Inoue

hirskino@gmail.com

Kakushi Matsushita

kks.matsushita@jiaikai.jp
Tadashi Matsumoto tmatsumoto@jiaikai.jp

Naomichi Arima

nao@m2.kufm.kagoshima-u.ac.jp

Kenji Ishitsuka

kenji-i@m.kufm.kagoshima-u.ac.jp

1 Department of Hematology, Izuro Imamura Hospital, 17-1 Horie, Kagoshima, Japan

2 Department of Hematology and Rheumatology, Kagoshima University Hospital, 8-35-1 Sakuragaoka, Kagoshima, Japan 